\title{
MicroRNA-720 inhibits pancreatic cancer cell proliferation and invasion by directly targeting cyclin D1
}

\author{
YU ZHANG ${ }^{1}$, YUEYING SU ${ }^{1}$, YABING ZHAO ${ }^{1}$, GUOQIANG LV $^{1}$ and YING LUO ${ }^{2}$ \\ ${ }^{1}$ Department of General Surgery, Yongcheng City People's Hospital, Yongcheng, Henan 476600; \\ ${ }^{2}$ Department of Hepatobiliary Surgery, Chinese PLA General Hospital, Beijing 100853, P.R. China
}

Received March 26, 2017; Accepted September 15, 2017

DOI: $10.3892 / \mathrm{mmr} .2017 .7732$

\begin{abstract}
Pancreatic cancer is the fourth leading cause of cancer-associated deaths in Western countries, and ranks sixth among cancer-associated diseases, with the highest mortality rate in China. Deregulation of micro (miR) RNA may contribute to the occurrence and progression of numerous cancers, including pancreatic cancer. In particular, deregulation of microRNA-720 (miR-720) has been reported in various types of human cancer. However, the expression and biological role of miR-720 in pancreatic cancer remains to be elucidated. The present study aimed to investigate the expression and functional role of miR-720 in pancreatic cancer and determine the underlying regulatory mechanism. The results demonstrated that miR-720 was expressed at low levels in pancreatic cancer tissue samples and cell lines. Upregulating miR-720 suppressed pancreatic cancer cell proliferation and invasion in vitro. Additionally, cyclin D1 (CCND1) was identified as the direct target gene of miR-720 in pancreatic cancer. Furthermore, CCND1 was significantly upregulated in pancreatic cancer tissues and inversely correlated with miR-720 expression. Furthermore, CCND1 re-expression partially abrogated the inhibitory effects of miR-720 on pancreatic cancer cells. Overall, miR-720 may act as a tumor suppressor by directly targeting CCND1 in pancreatic cancer.
\end{abstract}

\section{Introduction}

Pancreatic cancer is the fourth leading cause of cancer-related deaths in Western countries (1) and ranks sixth among cancer-related diseases with the highest mortality rate in China (2). Approximately 39,590 and 227,000 deaths due to pancreatic cancer are recorded annually in the USA and worldwide, respectively (3). Pancreatic ductal adenocarcinoma, the

Correspondence to: Professor Ying Luo, Department of Hepatobiliary Surgery, Chinese PLA General Hospital, 28 Fuxing Road, Beijing 100853, P.R. China

E-mail: luoying_ly@yeah.net

Key words: microRNA-720, pancreatic cancer, cyclin D1, proliferation, invasion most common type of pancreatic cancer, accounts for more than $85 \%$ of all pancreatic cancer cases (4). Currently, surgery resection is the primary treatment for long-term survival of patients with pancreatic cancer. However, only approximately 10-20\% of pancreatic cancer cases are suitable for surgery at the time of diagnosis because of difficulty in early diagnosis, local aggression and rapid progression (5). Despite considerable efforts to improve early detection and treatments for patients with pancreatic cancer, the overall prognosis remains unsatisfactory with 5-year survival rate of lower than 10\% (6-8). Moreover, the causes of pancreatic cancer remain unknown, although our knowledge of pancreatic cancer biology has progressed (9). Therefore, the mechanisms underlying pancreatic cancer formation and progression must be elucidated to develop effective therapeutic strategies for patients with this malignancy.

MicroRNAs (miRNAs) constitute a group of endogenous, noncoding and approximately 20-22 nucleotide-long RNAs that function as post-transcriptional regulators (10). MiRNAs modulate messenger RNA (mRNA) expression by base pairing to complementary sites in the 3'-untranslated regions (3'-UTRs) of target mRNAs, thereby inducing the degradation of the target mRNAs or inhibiting the translation of the target mRNAs (11). MiRNAs regulate over $30 \%$ coding genes (11). Moreover, miRNAs participate in various physiological processes, including cell proliferation, cycle, apoptosis, differentiation, metabolism and development of cells and pathological conditions, such as cardiovascular diseases, neurological diseases and tumours (12-14). Studies have reported that miRNAs are aberrantly expressed in various kinds of human cancers, such as pancreatic cancer (15), colorectal cancer (16), lung cancer (17) and bladder cancer (18). Depending on the characteristic of their target mRNAs, miRNAs may function as either oncogenes or tumour suppressors and thus play important roles in the occurrence and development of cancer (19). Therefore, the expression and roles of miRNAs in tumourigenesis and tumour development must be determined to elucidate the formation and progression of human cancers and improve the diagnosis and therapy of cancers.

Deregulation of miR-720 deregulation has been reported in several types of human cancers (20-22). Nevertheless, no specific studies have been conducted to reveal the expression and biological roles of miR-720 in pancreatic cancer. The present study aims to investigate the expression and roles of 
miR-720 in pancreatic cancer and determine the underlying regulatory mechanism.

\section{Material and methods}

Tissue samples. The present study was approved by the Ethics Committee of Yongcheng City People's Hospital (Ethics approval number: 20140037). Written informed consent was obtained from all pancreatic cancer patients enrolled in this research. Twenty-three pairs of pancreatic cancer tissues and matched adjacent normal pancreatic tissues were obtained from patients who received surgery resection at Yongcheng City People's Hospital between June 2014 to February 2016. All tissues were obtained from patients prior to chemotherapy or radiation therapy. Tissues were immediately frozen and stored at $-80^{\circ} \mathrm{C}$ prior to further processed.

Cell lines and cell culture. Human pancreatic cancer cell lines, Panc-1, Bxpc-3, Sw1990, and Aspc-1, were purchased from Cell Bank of Type Culture Collection of Chinese Academy of Sciences, Shanghai Institute of Cell Biology (Shanghai, China). The human normal pancreatic cell line (HPDE6c7) were obtained from American Type Culture Collection (Manassas, VA, USA). All cells were grown in Dulbecco's modified Eagle's medium (DMEM; Gibco, Thermo Scientific, Shanghai, China) supplemented with $10 \%$ fetal bovine serum (FBS; Gibco, Thermo Scientific, Shanghai, China), $100 \mathrm{U} / \mathrm{ml}$ penicillin and $100 \mathrm{U} / \mathrm{ml}$ streptomycin, and maintained at $37^{\circ} \mathrm{C}$ in a humidified incubator containing $5 \% \mathrm{CO}_{2}$.

Transfection. The miR-720 mimic and miRNA mimic negative control (NC) were purchased from Shanghai GenePharma Co., Ltd. (Shanghai, China). CCND1 expression vector (pcDNA 3.1-CCND1) and pcDNA3.1 empty vector were synthesized by Guangzhou RiboBio Co., Ltd. (Guangzhou, China). For transfection, cells were seeded into 6-well plates and cultured until reaching 70-80\% confluence. Cell transfection was performed using Lipofectamine 2000 (Invitrogen, Grand Island, NY, USA) according to the manufacturer's protocol. After incubation at $37^{\circ} \mathrm{C}$ with $5 \% \mathrm{CO}_{2}$ for $6 \mathrm{~h}$, the medium was replaced with DMEM containing $10 \%$ FBS.

RNA isolation and reverse transcription-quantitative polymerase chain reaction ( $R T-q P C R)$. Total RNA was isolated from tissues or cells using TRIzol reagent (Thermo Fisher Scientific, Waltham, MA, USA) according to the manufacturer's instructions. For miR-720 expression analysis, reverse transcription was performed using the TaqMan MicroRNA Reverse Transcription kit (Applied Biosystems, Foster City, CA, USA). MiR-720 expression was examined using the TaqMan MicroRNA PCR kit (Applied Biosystems, Foster City, CA, USA) on an ABI 7300 PCR Thermal Cycler (Thermo Fisher Scientific, Waltham, MA, USA). U6 served as an internal reference for miR-720 expression level. To quantify CCND1 mRNA level, total RNA was converted into cDNA using a PrimeScript RT Reagent kit (Takara Biotechnology, Co., Ltd., Dalian, China). Quantitative PCR was then performed to examine CCND1 mRNA expression using the Power SYBR Green PCR Master Mix (Thermo Fisher Scientific, Waltham, MA, USA). GAPDH was used as an internal control for CCND1
mRNA. The sequences of the primers used for PCR were as follows: miR-720 forward, 5'-GCGTGCTCTCGCTGGGG-3' and reverse, 5'-GTGCAGGGTCCGAGGT-3'; U6 forward, 5'-CTCGCTTCGGCAGCACA-3' and reverse, 5'-AACGCT TCACGAATTTGCGT-3'; CCND1 forward, 5'-CGGAGG ACAACAAACAGATC-3' and reverse, 5'-GGGTGTGCA AGCCAGGTCCA-3'; and GAPDH forward, 5'-ACAACTTTG GTATCGTGGAAGG-3' and reverse, 5'-GCCATCACGCCA CAGTTTC-3'. Each sample was performed in triplicate and relative expression was analyzed using the $2^{-\Delta \Delta \mathrm{Ct}}$ method (23).

Cell counting kit 8 (CCK 8) assay. CCK8 (Dojindo Laboratories, Kumamoto, Japan) assay was used to determine cell proliferation according to the manufacturers instructions. In brief, cells were plated into 96 -well plates at a density of $3 \times 10^{3}$ cells per well. Cells were then transfected with miRNA mimics or plasmid, and incubated at $37^{\circ} \mathrm{C}$ with $5 \% \mathrm{CO}_{2}$ for $0,24,48$, or $72 \mathrm{~h}$. At every time point, $10 \mu \mathrm{l}$ CCK 8 regent was added to the medium and the cells were incubated for a further $4 \mathrm{~h}$. Following incubation, the absorbance at a wavelength of $450 \mathrm{~nm}$ was detected using an enzyme-linked immunosorbent assay reader (Bio-Rad Laboratories, Inc., Hercules, CA, USA). Three independent experiments were performed with three replicates in each.

Matrigel invasion assay. Cell invasion ability was determined using a 24-well Matrigel-coated Transwell chamber with an $8-\mu \mathrm{m}$ pore-size polycarbonate membrane (BD Biosciences, San Jose, CA, USA). After incubation 48 h, 1x10 $10^{5}$ transfected cells in $200 \mu \mathrm{l}$ FBS-free DMEM were seeded into the upper chamber, after which $500 \mu 1$ DMEM with $10 \%$ FBS was added into the lower chamber. Cells were then incubated at $37^{\circ} \mathrm{C}$ with $5 \% \mathrm{CO}_{2}$ for $20 \mathrm{~h}$. Subsequently, cells on the upper side of the membranes were removed slightly by the dry cotton swabs. Invasive cells were fixed in methanol, stained with $0.5 \%$ crystal violet, photographed and counted in five representative microscopic fields under a light microscope (Olympus IX53; Olympus, Tokyo, Japan).

Bioinformatic analysis. Target genes of miR-720 were searched using the TargetScan (http//www.targetscan.org) and miRBase (http://www.mirbase.org/).

Luciferase reporter assay. The targeting relationship between miR-720 and 3'-UTR of CCND1 was detected by using luciferase reporter assay. Luciferase reporter vector containing the wild-type (pMIR-CCND1-3'-UTR WT) or mutant (pMIR-CCND1-3'-UTR MUT) 3'-UTR of CCND1 were synthesized by GenePharma Co., Ltd. Cells were seeded into 24-well plates at a density of $60-70 \%$ confluence and then co-transfected with the pMIR-CCND1-3'-UTR WT or pMIR-CCND1-3'-UTR MUT and miR-720 mimics or NC by using Lipofectamine 2000. $48 \mathrm{~h}$ after co-transfection, cells were harvested and luciferase activities were detected using the Dual-Luciferase assay system (Promega, Madison, WI, USA), according to the manufacturer's protocol. Firefly luciferase activities were normalized to Renilla luciferase activities. Each assay was performed as three replicates.

Western blotting. Total protein were extracted from tissues or cells using radioimmunoprecipitation assay lysis buffer 
(Beyotime Institute of Biotechnology, Haimen, China) supplemented with a protease inhibitor cocktail (Sigma-Aldrich, St. Louis, MO, USA). Protein concentration was examined using a BCA Protein Assay kit (Beyotime Biotechnology, Haimen, China). Equal quantities of protein were separated by $10 \%$ SDS-PAGE and electrotransferred onto PVDF membranes (EMD Millipore, Billerica, MA, USA). Subsequently, the membranes were blocked by $5 \%$ non-fat milk in Tris-based saline-Tween 20 (TBST) for $1 \mathrm{~h}$ at room temperature and blotted with primary antibodies: mouse anti-human CCND1 monoclonal antibody (sc-450; 1:1,000 dilution; Santa Cruz Biotechnology, CA, USA) or mouse anti-human GAPDH monoclonal antibody (sc-47724; 1:1,000 dilution; Santa Cruz Biotechnology, CA, USA). After washing thrice in TBST for $5 \mathrm{~min}$, the membranes were incubated with a corresponding horseradish peroxidase-conjugated secondary antibody (sc-2005; 1:5,000 dilution; Santa Cruz Biotechnology, CA, USA) at room temperature for $1 \mathrm{~h}$. Finally, proteins were detected by enhanced chemiluminescence (ECL) using a Pierce $^{\mathrm{TM}}$ ECL Western Blotting detection system (Thermo Fisher Scientific, Inc., Rockford, IL, USA), and the protein intensities were quantified using Quantity One software (Bio-Rad Laboratories, Inc., Hercules, CA, USA).

Statistical analysis. All statistical analyses were performed using student's t test or one way ANOVA test with SPSS software (version 16; SPSS, Inc., Chicago, IL, USA). Data are presented as mean \pm standard deviation. $P$ value less than 0.05 were considered significant.

\section{Results}

miR-720 expression is downregulated in pancreatic cancer tissues and cell lines. RT-qPCR analysis was performed in twenty-three pairs of pancreatic cancer tissues and matched adjacent normal pancreatic tissues to determine miR-720 expression. The results showed that miR-720 was significantly downregulated in pancreatic cancer tissues compared with that in their matched adjacent normal pancreatic tissues (Fig. 1A; $\mathrm{P}<0.05)$. Furthermore, miR-720 expression was detected in pancreatic cancer cell lines (Panc-1, Bxpc-3, Sw1990, Aspc-1) and a human normal pancreatic cell line (HPDE6c7). As shown in Fig. 1B, the expression levels of miR-720 decreased in pancreatic cancer cell lines than that in HPDE6c7 cells $(\mathrm{P}<0.05)$. The expression of miR-720 significantly decreased in Panc-1 and Sw1990 cell lines, which were then selected for subsequent experiments.

miR-720 inhibits the proliferation and invasion of pancreatic cancer cells. To delineate the potential roles of miR-720 in pancreatic cancer, we firstly transfected miR-720 mimics into Panc-1 and Sw1990 cells, which expressed relatively low miR-720 expression among four examined pancreatic cancer cell lines. RT-qPCR analysis was conducted to determine transfection efficiency. The results showed that miR-720 was markedly upregulated in Panc-1 and Sw1990 cells after transfection with miR-720 mimics (Fig. 2A; P<0.05). CCK8 assay was used to examine the effect of miR-720 overexpression on pancreatic cancer cell proliferation. As shown in Fig. 2B, upregulating miR-720 reduced the proliferation of
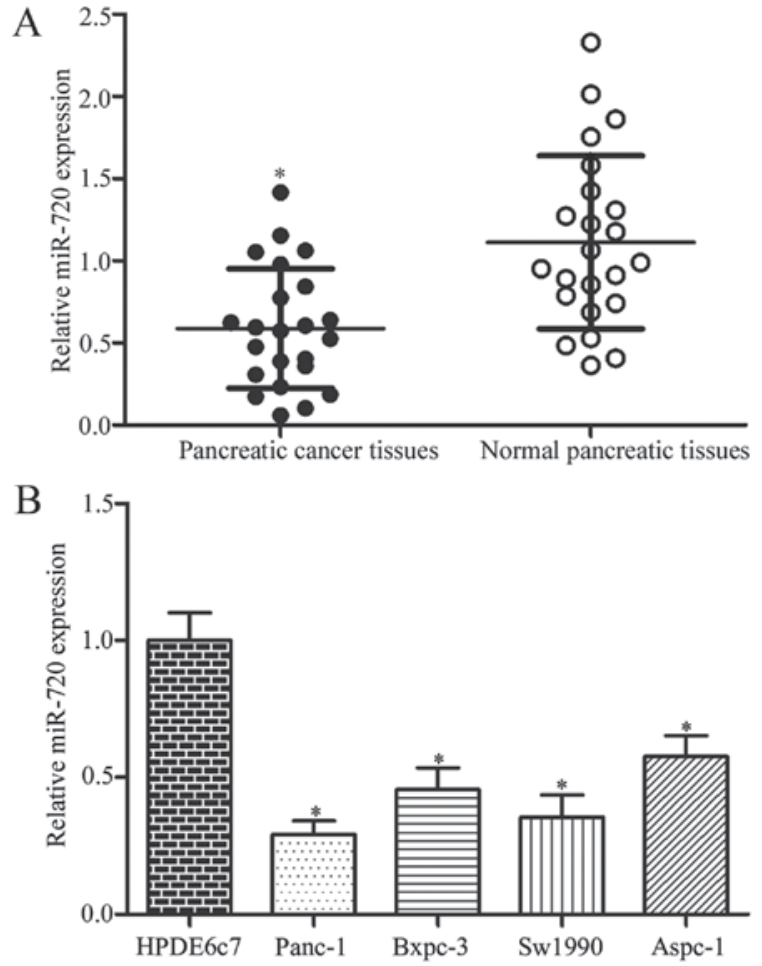

Figure 1. MiR-720 is lowly expressed in pancreatic cancer tissues and cell lines. (A) MiR-720 expression in pancreatic cancer tissues and matched adjacent normal pancreatic tissues was detected by RT-qPCR. ${ }^{*} \mathrm{P}<0.05$ compared with adjacent normal pancreatic tissues. (B) RT-qPCR analysis was conducted to examine miR-720 expression in pancreatic cancer cell lines (Panc-1, Bxpc-3, Sw1990, Aspc-1) and a human normal pancreatic cell line (HPDE6c7). ${ }^{*} \mathrm{P}<0.05$ compared with HPDE6c7.

Panc-1 and Sw1990 cells $(\mathrm{P}<0.05)$. Moreover, we performed Matrigel invasion assay to assess the effect of miR-720 on the invasion capacity of pancreatic cancer. Restoring the miR-720 expression reduced the invasive capability in both Panc-1 and Sw1990 cells (Fig. 2C; P<0.05). Hence, miR-720 may function as a tumour suppressor in pancreatic cancer.

miR-720 directly targets and mediates CCNDI expression. To investigate the molecular basis of miR-720 in regulating pancreatic cancer, we screened the potential targets of miR-720 by using bioinformatics analysis. We focused on CCND1 as putative target (Fig. 3A) because it plays important roles in pancreatic cancer initiation and progression (24-26). Luciferase reporter assay was performed in Panc-1 and Sw1990 cells transfected with miR-720 mimics or NC, along with pMIR-CCND1-3'-UTR WT or pMIR-CCND1-3'-UTR MUT, to determine whether or not CCND1 is a direct target of miR-720. As shown in Fig. 3B, miR-720 introduction decreased the luciferase activity of the wild-type CCND1 3'-UTR $(\mathrm{P}<0.05)$ but did not change of the activity of the mutant CCND1 3'-UTR.

To determine whether miR-720 could regulate endogenous CCND1 expression, we measured CCND1 mRNA and protein expression levels in Panc-1 and Sw1990 cells transfected with miR-720 mimics or NC. miR-720 overexpressing reduced the CCND1 expression in Panc-1 and Sw1990 cells at both mRNA (Fig. 3C; P<0.05) and protein (Fig. 3D; P<0.05) levels. Hence, miR-720 directly targets the 3'-UTR of CCND1 and 

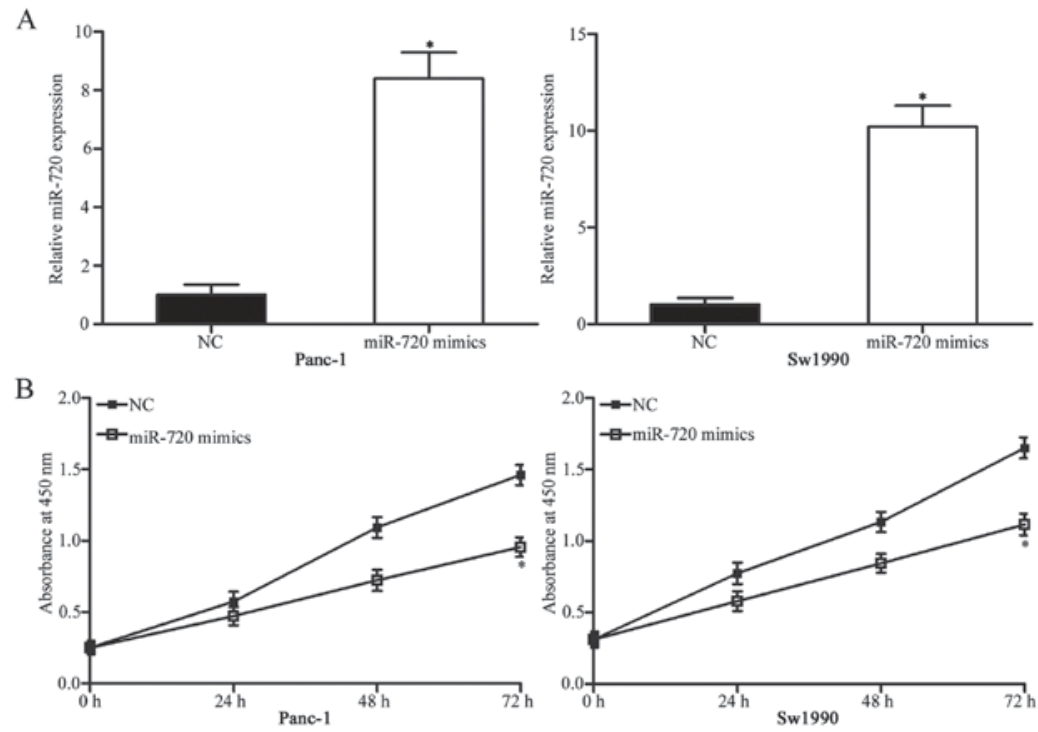

$\mathrm{C}$
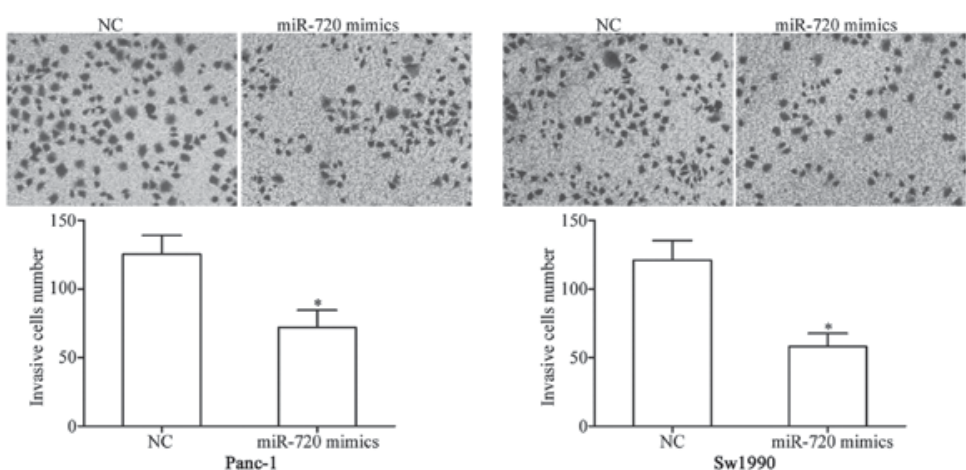

Figure 2. Effects of miR-720 overexpression on the proliferation and invasion of pancreatic cancer cells. (A) Panc-1 and Sw1990 cells were transfected with miR-720 mimics or NC. RT-qPCR analysis was conducted to evaluate endogenous miR-720 expression. "P<0.05 compared with NC. (B) CCK8 assay showed that miR-720 re-expression inhibited the proliferation of Panc-1 and Sw1990 cells. "P<0.05 compared with NC. (C) Matrigel invasion assay was conducted in Panc-1 and Sw1990 cells following transfection with miR-720 mimics or NC. Increasing the expression of miR-720 decreased the cell invasion capacities of Panc-1 and Sw1990 cells. "P<0.05 compared with NC.
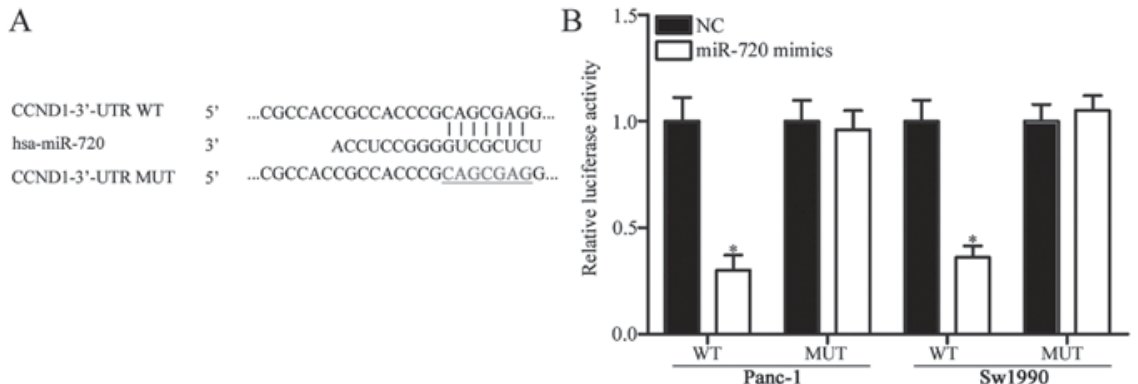

$\mathrm{C}$
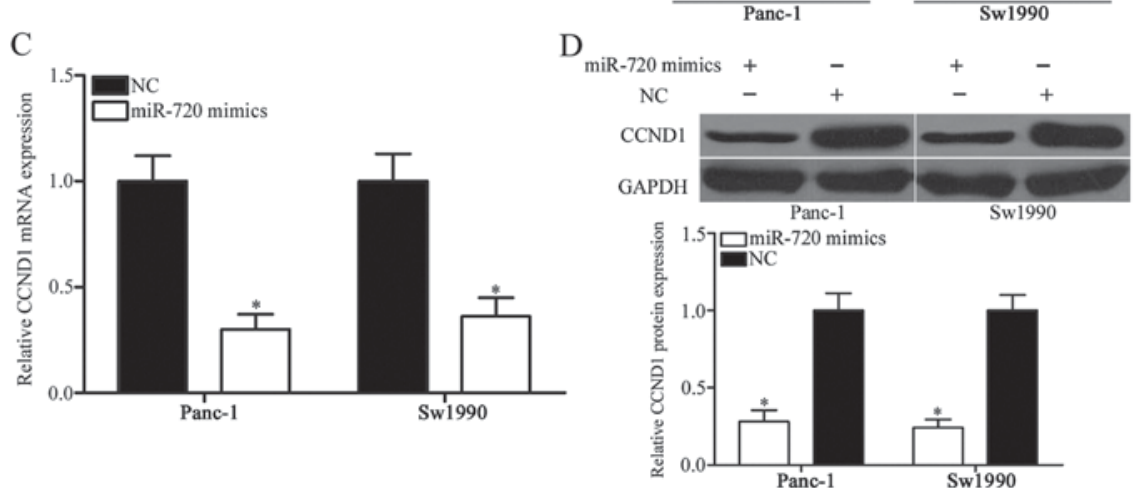

Figure 3. CCND1 is a direct target of miR-720 in pancreatic cancer. (A) Wild type and mutant of putative miR-720 binding sites in the 3'-untranslated region (3'-UTR) of CCND1. (B) Relative luciferase activities in Panc-1 and Sw1990 cells transfected with miR-720 mimics or NC, together with pMIR-CCND1-3'-UTR WT or pMIR-CCND1-3'-UTR MUT. "P<0.05 compared with NC. (C and D) RT-qPCR and Western blot analyses showed that miR-720 overexpression decreased CCND1 mRNA and protein expression levels in Panc-1 and Sw1990 cells. ${ }^{*} \mathrm{P}<0.05$ compared with NC. 
downregulates CCND1 mRNA and protein expression levels in pancreatic cancer cells.

CCND1 expression is upregulated and negatively related to miR-720 expression in pancreatic cancer. To further explore the functional significance of miR-720 in pancreatic cancer, we evaluated its association with CCND1 in pancreatic cancer tissues. RT-qPCR analysis was performed to quantify CCND1 mRNA expression in 23 pairs of pancreatic cancer tissues and matched adjacent normal pancreatic tissues. CCND1 mRNA expression increased in pancreatic cancer tissues compared with that in the adjacent normal pancreatic tissues (Fig. 4A; $\mathrm{P}<0.05$ ). In addition, Western blot analysis indicated that the protein expression level of CCND1 was significantly higher in pancreatic cancer tissues than that in the adjacent normal pancreatic tissues (Fig. 4B; P<0.05). Furthermore, Spearman's correlation analysis revealed that CCND1 mRNA was inversely correlated with miR-720 expression in pancreatic cancer tissues (Fig. 4C; $r=-0.6105, \mathrm{P}=0.0020$ ).

CCND1 reverses the tumour suppressive roles of $m i R-720$ in pancreatic cancer. To further identify whether the tumour-suppressing roles of miR-720 in pancreatic cancer cell proliferation and invasion were mediated by CCND1, we performed rescue experiments by introducing pcDNA 3.1-CCND1 in the presence or absence of miR-720 mimics in Panc-1 and Sw1990 cells. As shown in Fig. 5A, the ectopic expression of miR-720-induced downregulation of CCND1 was rescued by transfection of pcDNA 3.1-CCND1 $(\mathrm{P}<0.05)$. In addition, the increased expression of CCND1 could partially abrogate the inhibitory effects of miR-720 on the proliferation and invasion of pancreatic cancer cells (Fig. 5B and C; $\mathrm{P}<0.05)$. These findings suggested that miR-720 serves as a tumour suppressor in pancreatic cancer, at least in part, by negatively regulating CCND1.

\section{Discussion}

Deregulation of miRNA might contribute to the occurrence and progression of many cancer types, including pancreatic cancer (27-29). A large number of miRNAs play crucial roles in the proliferation, apoptosis, cycle, metastasis and angiogenesis of pancreatic cancer cells; hence, suggesting that miRNA could be used in pancreatic cancer diagnosis and treatment (30-32). In the present study, miR-720 was downregulated in pancreatic cancer tissues and cell lines. Resumption of miR-720 expression suppressed the proliferation and invasion of pancreatic cancer cells in vitro. Furthermore, CCND1 was validated as a direct and functional target of miR-720 in pancreatic cancer. These results indicated that miR-720 acts as a tumour suppressor in pancreatic cancer and thus can be used to treat patients with pancreatic cancer.

Dysregulation of miR-720 has been reported in several types of human cancer. For example, miR-720 expression decreased in breast cancer tissues and was found to be significantly correlated with lymph node metastasis (20). A previous study indicated that miR-720 was upregulated in cervical cancer tissues compared with that in normal cervical tissues (21). In colorectal cancer, the expression levels of miR-720 was higher in tumour tissues than that in corresponding normal-appearing
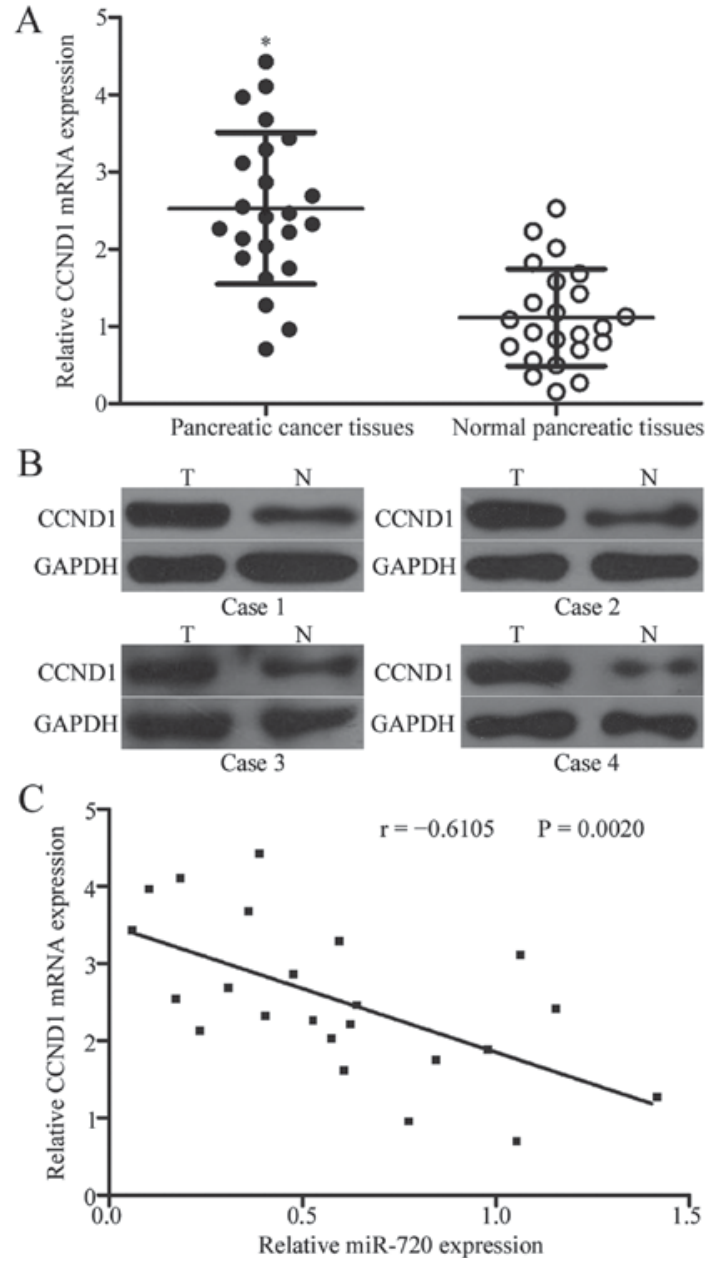

Figure 4. Inverse correlation between miR-720 and CCND1 in pancreatic cancer tissues. (A and B) MRNA and protein expression levels of CCND1 in pancreatic cancer tissues and matched adjacent normal pancreatic tissues were determined using RT-qPCR and Western blot analyses. ${ }^{*} \mathrm{P}<0.05$ compared with adjacent normal pancreatic tissues. $\mathrm{T}$, pancreatic cancer tissues; N, adjacent normal pancreatic tissues. (C) Evaluation of the inverse correlation between miR-720 and CCND1 in pancreatic cancer tissues by Spearman's correlation analysis $(\mathrm{r}=-0.6105, \mathrm{P}=0.0020)$.

tissues. High miR-720 expression is correlated with tumour size, tumour-node-metastasis stage, lymphatic metastasis and distant metastasis and could lead to poor 5-year overall survival rate in patients with colorectal cancer (22). Additionally, miR-720 expression increased remarkably in the serum of patients with colorectal cancer compared with that in healthy patients. The expression level of miR-720 is also associated with male gender and lymph node metastasis in patients with colorectal cancer. Thus, miR-720 expression exhibits tissue specificity, and may be a prognostic marker in these specific types of cancer.

Several studies have identified the functions of miR-720 in cancer progression. For instance, miR-720 served as a tumour suppressor in breast cancer by inhibiting tumour cell metastasis both in vitro and in vivo (20). Nevertheless, miR-720 was validated as an oncogene in tumourigenesis and development. Tang et al reported that restoration expression of miR-720 decreased E-cadherin expression, increased vimentin expression and promoted cervical cancer cell migration (21). Wang et al found that miR-720 overexpression promoted cell proliferation, colony formation ability and metastasis of 


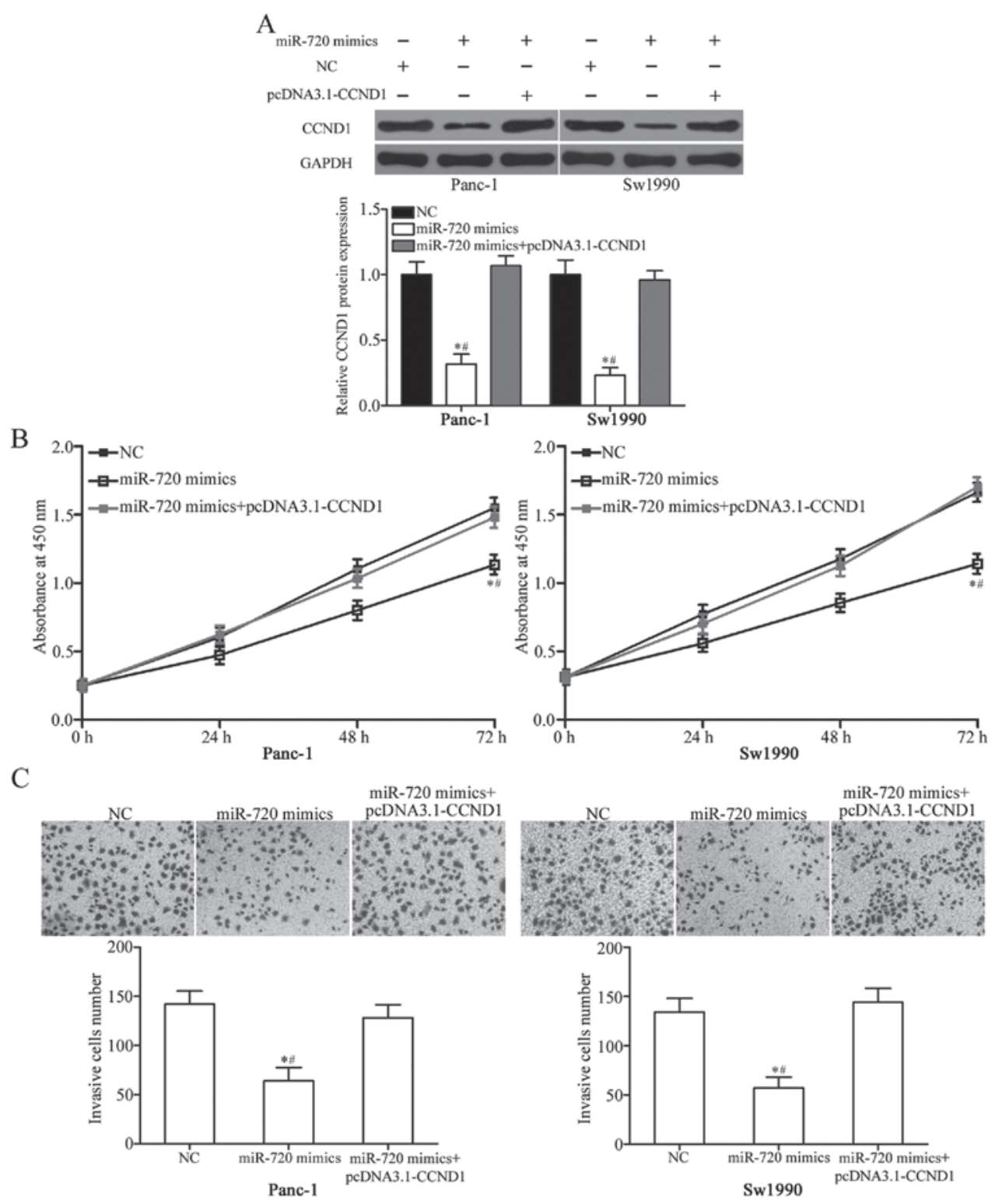

Figure 5. Upregulation of CCND1 prevents the inhibitory effects of miR-720 on pancreatic cancer cells. (A) Protein expression of CCND1 in Panc-1 and Sw1990 cells after transfection with miR-720 mimics, NC or miR-720 mimics, along with pcDNA 3.1-CCND1. "P<0.05 compared with NC and miR-720 mimics + pcDNA 3.1-CCND1. (B and C) CCK8 assay and Matrigel invasion assay showed that CCND1 upregulation partly reversed the suppressive effects of miR-720 on the proliferation and invasion of Panc-1 and Sw1990 cells. " $\mathrm{P}<0.05$ compared with NC and miR-720 mimics + pcDNA 3.1-CCND1.

colorectal cancer (22). These conflicting findings indicate that miR-720 exhibits tissue specificity, which could be explained by the non-ideal complementarity of the interactions between miRNA and their direct target genes.

The targets of miR-720 include TWIST1 (20) in breast cancer, Rab35 (21) in cervical cancer, StarD13 in colorectal cancer (22) and GATA3 in macrophages (33). The present study demonstrated that CCND1 is a direct functional target of miR-720 in pancreatic cancer, as supported through several lines of evidence. Firstly, bioinformatics analysis predicted that CCND1 is a potential target of miR-720. Secondly, miR-720 overexpression decreased CCND1 3'-UTR luciferase activity, an effect abolished by the mutation of the miR-720 seed region. Thirdly, miR-720 negatively regulated CCND1 expression in pancreatic cancer cells at mRNA and protein levels. Furthermore, CCND1 was upregulated in pancreatic cancer tissues and inversely correlated with miR-720 expression. The enforced expression of CCND1 could also partially abrogate the inhibitory effects of miR-720 on pancreatic cancer cells. These results demonstrate that CCND1 is a direct target gene of miR-720 in pancreatic cancer.

The CCND1 gene, located on chromosome 11q13, is a well-known oncogene that is frequently overexpressed in various kinds of human cancers, such as breast cancer (34), lung cancer (35), gastric cancer (36) and bladder cancer (37). In pancreatic cancer, CCND1 was overexpressed in tumour tissues; the expression of this gene is significantly correlated with extent of differentiation and poor prognosis $(38,39)$. CCND1 also plays important roles in pancreatic cancer formation and progression. Functional assays demonstrated that CCND1 knockdown inhibited cell proliferation, soft agar colony formation, metastasis, metabolism in vitro and cell growth in vivo (24-26). Moreover, CCND1 underexpression increased the chemosensitivity of pancreatic cancer cells to 
5-fluorouracil, 5-fluoro-2'-deoxyuridine and mitoxantrone by downregulating multiple chemoresistance genes (40). Hence, regulating miR-720/CCND1 axis could be a novel and effective therapeutic strategy for inhibiting the rapid growth and metastasis of pancreatic cancer.

In conclusion, miR-720 acted as a tumour suppressor in pancreatic cancer by directly targeting CCND1. The precise mechanism of miR-720/CCND1 axis in pancreatic cancer must be determined to elucidate the pathogenesis of pancreatic cancer and develop new treatment approach for patients with pancreatic cancer.

\section{References}

1. Torre LA, Bray F, Siegel RL, Ferlay J, Lortet-Tieulent J and Jemal A: Global cancer statistics, 2012. CA Cancer J Clin 65: 87-108, 2015

2. Chen W, Zheng R, Zhang S, Zhao P, Li G, Wu L and He J: Report of incidence and mortality in China cancer registries, 2009. Chin J Cancer Res 25: 10-21, 2013.

3. Siegel RL, Miller KD and Jemal A: Cancer statistics, 2015. CA Cancer J Clin 65: 5-29, 2015

4. Modolell I, Guarner L and Malagelada JR: Vagaries of clinica presentation of pancreatic and biliary tract cancer. Ann Oncol 10 (Suppl 4): S82-S84, 1999.

5. Cartwright T, Richards DA and Boehm KA: Cancer of the pancreas: Are we making progress? A review of studies in the US Oncology Research Network. Cancer Control 15: 308-313, 2008

6. Paulson AS, Tran Cao HS, Tempero MA and Lowy AM: Therapeutic advances in pancreatic cancer. Gastroenterology 144: 1316-1326, 2013

7. Ansari D, Gustafsson A and Andersson R: Update on the management of pancreatic cancer: Surgery is not enough. World J Gastroenterol 21: 3157-3165, 2015.

8. Puleo F, Maréchal R, Demetter P, Bali MA, Calomme A, Closset J, Bachet JB, Deviere J and Van Laethem JL: New challenges in perioperative management of pancreatic cancer. World J Gastroenterol 21: 2281-2293, 2015

9. Greer JB, Lynch HT and Brand RE: Hereditary pancreatic cancer: A clinical perspective. Best Pract Res Clin Gastroenterol 23 159-170, 2009.

10. Szafranska AE, Davison TS, John J, Cannon T, Sipos B, Maghnouj A, Labourier E and Hahn SA: MicroRNA expression alterations are linked to tumorigenesis and non-neoplastic processes in pancreatic ductal adenocarcinoma. Oncogene 26: 4442-4452, 2007.

11. Bartel DP: MicroRNAs: Target recognition and regulatory functions. Cell 136: 215-233, 2009.

12. Wang Q, Wei L, Guan X, Wu Y, Zou Q and Ji Z: Briefing in family characteristics of microRNAs and their applications in cancer research. Biochim Biophys Acta 1844: 191-197, 2014.

13. Li M, Fu W, Wo L, Shu X, Liu F and Li C: miR-128 and its target genes in tumorigenesis and metastasis. Exp Cell Res 319 3059-3064, 2013.

14. Kaplan BB, Kar AN, Gioio AE and Aschrafi A: MicroRNAs in the axon and presynaptic nerve terminal. Front Cell Neurosci 7: $126,2013$.

15. Chang W, Liu M, Xu J, Fu H, Zhou B, Yuan T and Chen P: MiR-377 inhibits the proliferation of pancreatic cancer by targeting Pim-3. Tumour Biol 37: 14813-14824, 2016.

16. Yoo HI, Kim BK and Yoon SK: MicroRNA-330-5p negatively regulates ITGA5 expression in human colorectal cancer. Oncol Rep 36: 3023-3029, 2016.

17. Zeng Y, Zhu J, Shen D, Qin H, Lei Z, Li W, Liu Z and Huang JA: MicroRNA-205 targets SMAD4 in non-small cell lung cancer and promotes lung cancer cell growth in vitro and in vivo. Oncotarget 8: 30817-30829, 2017.

18. Zhou Y, Wu D, Tao J, Qu P, Zhou Z and Hou J: MicroRNA-133 inhibits cell proliferation, migration and invasion by targeting epidermal growth factor receptor and its downstream effector proteins in bladder cancer. Scand J Urol 47: 423-432, 2013.

19. Bhardwaj A, Singh S and Singh AP: MicroRNA-based Cancer Therapeutics: Big Hope from Small RNAs. Mol Cell Pharmacol 2: 213-219, 2010.
20. Li LZ, Zhang CZ, Liu LL, Yi C, Lu SX, Zhou X, Zhang ZJ, Peng YH, Yang YZ and Yun JP: miR-720 inhibits tumor invasion and migration in breast cancer by targeting TWIST1. Carcinogenesis 35: 469-478, 2014

21. Tang Y, Lin Y, Li C, Hu X, Liu Y, He M, Luo J, Sun G, Wang T, Li W and Guo M: MicroRNA-720 promotes in vitro cell migration by targeting Rab35 expression in cervical cancer cells. Cell Biosci 5: 56, 2015.

22. Wang X, Kuang Y, Shen X, Zhou H, Chen Y, Han Y, Yuan B, Zhou J, Zhao H, Zhi Q and Xue X: Evaluation of miR-720 prognostic significance in patients with colorectal cancer. Tumour Biol 36: 719-727, 2015.

23. Livak KJ and Schmittgen TD: Analysis of relative gene expression data using real-time quantitative PCR and the 2(-Delta Delta C(T)) method. Methods 25: 402-408, 2001

24. Xu Y, Liu T and Gao J: Effects of antisense cyclin D1 expressing vector on the cell growth and apoptosis of pancreatic carcinoma. Zhonghua Bing Li Xue Za Zhi 27: 348-351, 1998 (In Chinese).

25. Yan L, Wang Y, Wang ZZ, Rong YT, Chen LL, Li Q, Liu T, Chen YH, Li YD, Huang ZH and Peng J: Cell motility and spreading promoted by CEACAM6 through cyclin D1/CDK4 in human pancreatic carcinoma. Oncol Rep 35: 418-426, 2016.

26. Lee Y, Ko D, Min HJ, Kim SB, Ahn HM, Lee Y and Kim S: TMPRSS4 induces invasion and proliferation of prostate cancer cells through induction of Slug and cyclin D1. Oncotarget 7: 50315-50332, 2016.

27. Mizuguchi Y, Takizawa T, Yoshida H and Uchida E: Dysregulated miRNA in progression of hepatocellular carcinoma: A systematic review. Hepatol Res 46: 391-406, 2016.

28. Liu H, Wang H, Liu X and Yu T: miR-1271 inhibits migration, invasion and epithelial-mesenchymal transition by targeting ZEB1 and TWIST1 in pancreatic cancer cells. Biochem Biophys Res Commun 472: 346-352, 2016.

29. Ren ZG, Dong SX, Han P and Qi J: miR-203 promotes proliferation, migration and invasion by degrading SIK1 in pancreatic cancer. Oncol Rep 35: 1365-1374, 2016.

30. Trehoux S, Lahdaoui F, Delpu Y, Renaud F, Leteurtre E, Torrisani J, Jonckheere N and Van Seuningen I: Micro-RNAs miR-29a and miR-330-5p function as tumor suppressors by targeting the MUC1 mucin in pancreatic cancer cells. Biochim Biophys Acta 1853: 2392-2403, 2015.

31. Xia X, Zhang K, Cen G, Jiang T, Cao J, Huang K, Huang C, Zhao Q and Qiu Z: MicroRNA-301a-3p promotes pancreatic cancer progression via negative regulation of SMAD4. Oncotarget 6: 21046-21063, 2015.

32. Li L, Li B, Chen D, Liu L, Huang C, Lu Z, Lun L and Wan X: miR-139 and miR-200c regulate pancreatic cancer endothelial cell migration and angiogenesis. Oncol Rep 34: 51-58, 2015.

33. Zhong Y and Yi C: MicroRNA-720 suppresses M2 macrophage polarization by targeting GATA3. Biosci Rep 36: pii: e00363, 2016.

34. Li X, Huo X, Li W, Yang Q, Wang Y and Kang X: Genetic association between cyclin D1 polymorphism and breast cancer susceptibility. Tumour Biol 35: 11959-11965, 2014.

35. Betticher DC, Heighway J, Hasleton PS, Altermatt HJ, Ryder WD, Cerny $\mathrm{T}$ and Thatcher N: Prognostic significance of CCND1 (cyclin D1) overexpression in primary resected non-small-cell lung cancer. Br J Cancer 73: 294-300, 1996.

36. Kumari S, Puneet, Prasad SB, Yadav SS, Kumar M, Khanna A, Dixit VK, Nath G, Singh S and Narayan G: Cyclin D1 and cyclin E2 are differentially expressed in gastric cancer. Med Oncol 33: 40, 2016.

37. Kopparapu PK, Boorjian SA, Robinson BD, Downes M, Gudas LJ, Mongan NP and Persson JL: Expression of cyclin d1 and its association with disease characteristics in bladder cancer. Anticancer Res 33: 5235-5242, 2013.

38. Gansauge S, Gansauge F, Ramadani M, Stobbe H, Rau B, Harada N and Beger HG: Overexpression of cyclin D1 in human pancreatic carcinoma is associated with poor prognosis. Cancer Res 57: 1634-1637, 1997.

39. Li YJ and Ji XR: Relationship between the expression of beta-cat, cyclin D1 and c-myc and the occurance and biological behavior of pancreatic cancer. Zhonghua Bing Li Xue Za Zhi 32: 238-241, 2003 (In Chinese).

40. Kornmann M, Danenberg KD, Arber N, Beger HG, Danenberg PV and Korc M: Inhibition of cyclin D1 expression in human pancreatic cancer cells is associated with increased chemosensitivity and decreased expression of multiple chemoresistance genes. Cancer Res 59: 3505-3511, 1999. 